\title{
Resolving Multilayer Structure of Pressure Sensitive Adhesive by Atomic Force Microscopy (AFM)
}

\author{
Hu Duan ${ }^{1}$, Shuang Qin ${ }^{1}$ \\ ${ }^{1}$ Materials Science and Characterization, Avery Dennison Corporation, Mentor, OH 44060, USA
}

Pressure sensitive adhesive (PSA) tape and label products are used in many important applications for both consumers and industrial users. A conventional pressure-sensitive adhesive (PSA) label construction comprises a laminate of a facestock, a pressure-sensitive adhesive layer, and a coated release liner. Recently with the new development of advanced manufacturing technology, multilayer adhesives can be simultaneously coated or coextruded onto the release liner using dual die or curtain coating technology. Such new multilayer PSA structure provides additional benefits including improvement of anchorage of removable adhesive, reduction of manufacturing cost, enhancement of converting performance, improvement of adhesion performance etc[1-3].

The performance of the PSA products with multilayer adhesive is closely related with the chemical compositions of the adhesives and layer structure of the adhesives. A good understanding of the structure, including the ratio of the thickness of each individual adhesive layer and the interface of the adhesive layers, is critical for development of cost effective high performance PSA products. However, in comparison with structural characterization of multilayer polymer film, it is more challenging to identify multilayer structure of adhesives because 1: the layers have very similar chemical compositions, usually random copolymers of 2-ethyl hexyl acrylate (or iso-octyl acrylate), butyl acrylate, and other acrylates, 2: the adhesive layers can be as thin as less than $5 \mu \mathrm{m}$ and the thickness can vary significantly on rough paper substrate, 3: the low Tg adhesive polymer and porous nature of the paper substrate impose additional difficulty to obtain thin section of smooth block face. Figure 1. shows a scanning electron microscopy (SEM) image of a known dual layer adhesive between paper substrates. There is no layer contrast or interfacial boundary between the two layers. Optical microscopy (OM) and transmission electron microscopy (TEM) suffer the same contrast issue as well. FTIR imaging and Raman imaging have also been attempted on the dual layer adhesives. It was not successful because the chemical compositions of the two adhesives are too similar to resolve.

While common conventional microscopy and spectroscopy techniques have difficulties to identify the structure of multilayer adhesive, we demonstrate that Atomic Force Microscopy (AFM) phase imaging can be a valuable tool to elucidate subtle structural difference between the adhesive layers. Film labels or tape samples can be cross sectioned by ultramicrotomy at $-30^{\circ} \mathrm{C}$. Paper labels can be cross sectioned with sharp razor on pre-cooled samples. The obtained block face was studied with AFM phase image mode. In this mode, the spatial variation in surface elasticity is detected by monitoring the phase shift associated with the probe's resonance and its proximal interaction with the sample. Figure 2 shows the adhesive morphology difference in a dual layer PSA product. Adhesive layer 1 is a permanent emulsion adhesive. It displays cell like structure with distinct cell boundaries. Such cell structure was formed due to incomplete fusion of the latex particle during coalescence stage. The bright network with high phase angle mainly consists of serum solids (mainly surfactant)[4-5]. Adhesive layer 2 is a removable PSA. In this layer, wax additives were intentionally added into this layer to reduce the adhesion. Spherical particles in the size of $100 \mathrm{~nm}$ to $1 \mu \mathrm{m}$ are distributed throughout the layer. An important industrial application of this imaging technique is to optimize the coating process parameters. Ideally, interfacial 
mixing is undesired because it will compromise the performance of each layer. When the viscosity, flowing behavior and drying speed are not optimized, interfacial instability and mixing will occur and negatively impact the performance of the product. Figure 3 shows such interfacial mixing under nonoptimized coating process. Adhesive 1 becomes instable at the interface and some of the droplets break up and mix into adhesive 2 .

\section{References:}

[1] L. Xie, et al, US patent 6,503,620 B1, 2003

[2] J Ercillo, et al, US patent 5,827,609, 1998

[3] Y Sasaki, et al, US patent 5,558,913, 1996

[4] G Xu, et al, J. Phys. Chem. B 113 (2009), p10189-10195

[5] CH Lei, et al, Journal of Colloid and Interface Science 307 (2007), p56-63

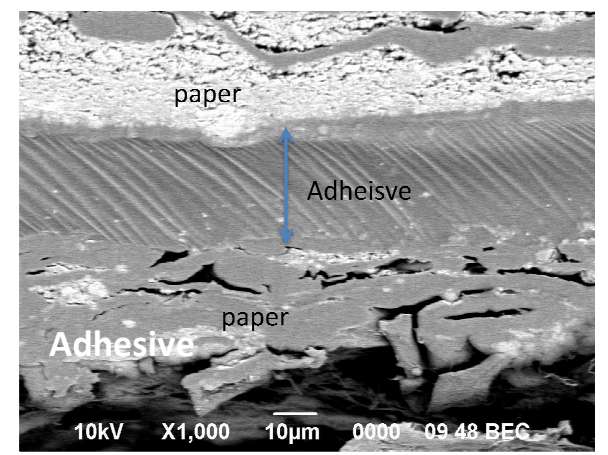

Figure 1. Scanning electron micrograph of a dual layer PSA product

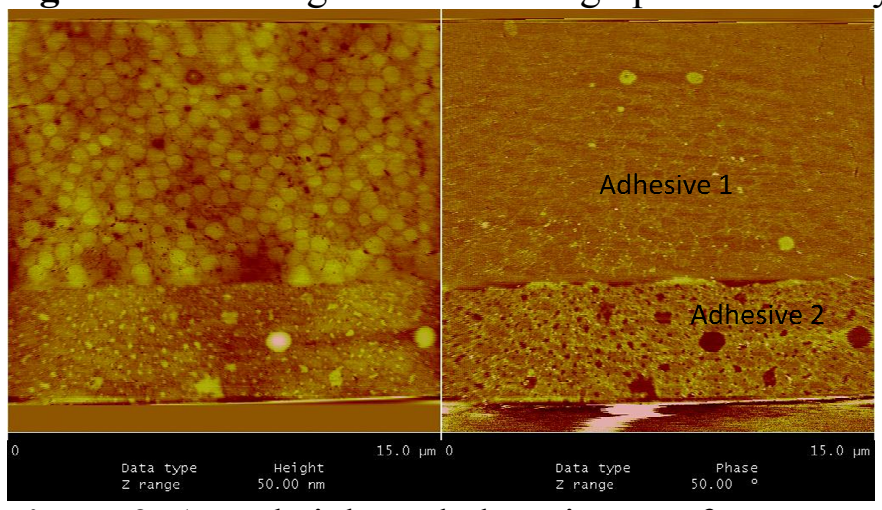

Figure 2. AFM height and phase image of a commercial dual layer PSA label. Scan size $15 X 15 \mu \mathrm{m}$.

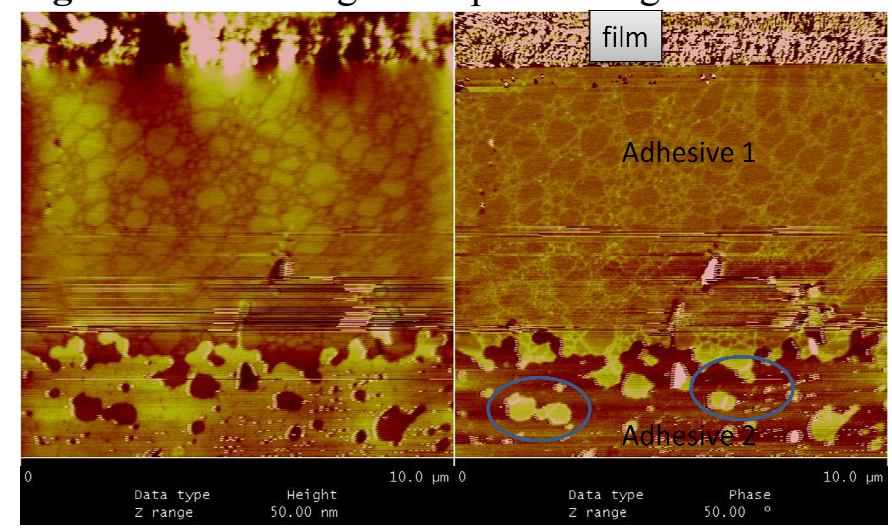

Figure 3. AFM height and phase image of a pilot trial dual layer PSA label. Scan size 10X10 $\mu \mathrm{m}$. 